\title{
Hábitos sexuais de mulheres com câncer ginecológico
}

\author{
Sexual habits of women with gynecological cancer \\ Hábitos sexuales de mujeres con cáncer ginecológico
}

Recebido: 24/01/2022 | Revisado: 29/01/2022 | Aceito: 01/02/2022 | Publicado: 03/02/2022

Felipe Cardozo Modesto

ORCID: https://orcid.org/0000-0001-9362-4231 Instituto Nacional de Câncer José Alencar Gomes da Silva, Brasil E-mail: felipe.modesto@inca.gov.br

Letícia Viana Chebabe Mendes Lattanzi

ORCID: https://orcid.org/0000-0003-1328-5853

Escola de Medicina Souza Marques, Brasil E-mail: leticiavcmlattanzi@gmail.com

Caroline Graça Mota Damasceno

ORCID: https://orcid.org/0000-0003-1216-0573

Escola de Medicina Souza Marques, Brasil E-mail: carolgracamd1@gmail.com

Marcela Arruda Karl

ORCID: https://orcid.org/0000-0003-3523-1792 Universidade Federal Fluminense, Brasil E-mail: celakarl@gmail.com

Raquel Boechat de Moura Carvalho

ORCID: https://orcid.org/0000-0003-0216-4669

Instituto Nacional de Câncer José Alencar Gomes da Silva, Brasil E-mail: raquel.boechat@inca.gov.br

Kamila Rodrigues Ferreira

ORCID: https://orcid.org/0000-0001-7655-7102 Instituto Nacional de Câncer José Alencar Gomes da Silva, Brasil E-mail: kamilafer.rj@gmail.com

Patrícia Lopes-Souza

ORCID; https://orcid org/0000-0003-0960-9245 Instituto Nacional de Câncer José Alencar Gomes da Silva, Brasil

E-mail: patricialopes.souza@yahoo.com.br

Natalia Beatriz Lima Pimentel

ORCID: https://orcid.org/0000-0003-1067-6840 Instituto Nacional de Câncer José Alencar Gomes da Silva, Brasil E-mail: nataliabeatriz@outlook.com

Adriana Maria de Oliveira

ORCID: https://orcid.org/0000-0002-9115-5803 Instituto Nacional de Câncer José Alencar Gomes da Silva, Brasil E-mail: dicaoliveira.1177@gmail.com

Karla Biancha Silva de Andrade

ORCID: https://orcid.org/0000-0002-6216-484X Instituto Nacional de Câncer José Alencar Gomes da Silva, Brasil E-mail: karla.biancha@gmail.com

\begin{abstract}
Resumo
Introdução: A progressão da doença e os efeitos colaterais dos tratamentos repercutem na vida das pacientes com câncer ginecológico. Ponderar sobre hábitos sexuais após diagnóstico pode facilitar entender fatores psicológicos e físicos que influenciam na sexualidade. Objetivos: Verificar os hábitos sexuais de mulheres com câncer ginecológico após diagnóstico e os fatores que impactam na sexualidade dessas mulheres. Métodos: Pacientes hospitalizadas >18 anos, com câncer ginecológico, responderam questionário com 16 questões fechadas, 11 sobre perfil clínico e 5 sobre

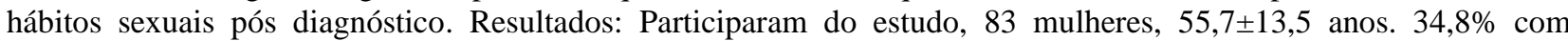
estadiamento >IIb. 77,11\% declaram não ter vida sexual ativa, estando 36,7\% atribuído à ausência de parceiro, $12,05 \%$ por dor, $16,87 \%$ por perda da libido e $13,25 \%$ por medo ou limitação clínica. $26,51 \%$ das mulheres referem impacto da sexualidade na sua qualidade de vida. Conclusão: A situação conjugal influencia na função sexual das mulheres, mas dor e perda de libido são fatores que mais impactam na sexualidade dessas mulheres. Maior exploração dessa temática faz-se necessário para melhor instruções das pacientes, acompanhantes e, sobretudo, dos profissionais de saúde, para elaboração de estratégias com foco na qualidade de vida.
\end{abstract}

Palavras-chave: Sexualidade; Câncer ginecológico; Hospitalização; Qualidade de vida; Saúde da mulher. 


\begin{abstract}
Introduction: The progression of the disease and the side effects of treatments affect the lives of patients with gynecological cancer. Pondering about sexual habits after diagnosis can make it easier to understand psychological and physical factors that influence sexuality. Objectives: To verify the sexual habits of women with gynecological cancer after diagnosis and the factors that impact the sexuality of these women. Methods: Hospitalized patients $>18$ years old, with gynecological cancer, answered a questionnaire with 16 closed questions, 11 about clinical profile and 5 about post-diagnosis sexual habits. Results: 83 women, $55.7 \pm 13.5$ years old, participated in the study. $34.8 \%$ with staging > IIb. $77.11 \%$ declared not having an active sexual life, $36.7 \%$ being attributed to the absence of a partner, $12.05 \%$ due to pain, $16.87 \%$ due to loss of libido and $13.25 \%$ due to fear or clinical limitation. $26.51 \%$ of women report the impact of sexuality on their quality of life. Conclusion: The marital status influences the sexual function of women, but pain and loss of libido are factors that most impact the sexuality of these women. Further exploration of this theme is necessary to better instruct patients, companions and, above all, health professionals, to develop strategies focused on quality of life.
\end{abstract}

Keywords: Sexuality; Gynecological cancer; Hospitalization; Quality of life; Women's health.

\title{
Resumen
}

Introducción: La progresión de la enfermedad y los efectos secundarios de los tratamientos afectan la vida de las pacientes con cáncer ginecológico. Reflexionar sobre los hábitos sexuales después del diagnóstico puede facilitar la comprensión de los factores psicológicos y físicos que influyen en la sexualidad. Objetivos: Verificar los hábitos sexuales de mujeres con cáncer ginecológico después del diagnóstico y los factores que impactan en la sexualidad de estas mujeres. Métodos: Pacientes hospitalizadas >18 años, con cáncer ginecológico, respondieron un cuestionario con 16 preguntas cerradas, 11 sobre perfil clínico y 5 sobre hábitos sexuales post-diagnóstico. Resultados: Participaron del estudio 83 mujeres, 55,7 $\pm 13,5$ años. 34,8\% con estadificación >IIb. El 77,11\% declaró no tener vida sexual activa, atribuyéndose el $36,7 \%$ a ausencia de pareja, el 12,05\% por dolor, el 16,87\% por pérdida de libido y el 13,25\% por miedo o limitación clínica. El 26,51\% de las mujeres reportan el impacto de la sexualidad en su calidad de vida. Conclusión: El estado civil influye en la función sexual de la mujer, pero el dolor y la pérdida de la libido son los factores que más impactan en la sexualidad de estas mujeres. Es necesaria una mayor exploración de este tema para instruir mejor a los pacientes, acompañantes y, sobre todo, a los profesionales de la salud, para desarrollar estrategias enfocadas en la calidad de vida.

Palabras clave: Sexualidad; Cáncer ginecológico; Hospitalización; Calidad de vida; Salud de la mujer.

\section{Introdução}

As neoplasias malignas ginecológicas compreendem um grupo de câncer em diferentes órgãos do aparelho genital feminino, tais como os tumores de colo do útero, cânceres do corpo do útero, ovário, vulva e vagina. Nesse grupo de doenças, merece destaque o câncer de colo de útero, o qual é o $3^{\circ}$ mais frequente entre as mulheres no Brasil, o câncer de ovário e o câncer de endométrio (corpo do útero), ocupando a $7^{\mathrm{a}}$ e $8^{\mathrm{a}}$ posição na incidência nacional de neoplasia maligna na população feminina brasileira, respectivamente (Inca, 2020).

A medicina avançou consideravelmente no que se refere ao tratamento do câncer ginecológico, sendo a cirurgia, a radioterapia, a quimioterapia, a hormonioterapia ou o uso dessas terapêuticas de forma combinada, as principais formas de terapia antineoplásicas disponíveis. Entretanto, a opção ideal de tratamento para esse câncer deve ser ponderada diante de cada caso, devendo, portanto, considerar as características da paciente, sua individualidade, a fisiopatologia e o estadiamento da doença (American Cancer Society, 2019).

Além da própria progressão do câncer ginecológico, os efeitos colaterais dos tratamentos antineoplásicos podem causar grandes repercussões na qualidade vida das pacientes portadoras dessa enfermidade, pois se trata de uma doença que afeta órgãos simbólicos, principalmente na cultura brasileira, especialmente por se associarem a esfera da sexualidade (Correia et al, 2018).

No âmbito da qualidade de vida relacionada à saúde, mostra-se relevante ponderar sobre a sexualidade destas pacientes antes e após o tratamento. Os efeitos adversos físicos advindos do plano terapêutico podem provocar repercussões que impactam diretamente na vida dessas mulheres, tanto físico como psicologicamente, afetando o comportamento sexual e podem comprometer a qualidade de sua vida social, conjugal, mudanças na autoimagem, perda da libido e baixa autoestima (Correia et al, 2018). 
Paralelo a esse entendimento, a sexualidade é uma importante preocupação para muitas mulheres após o diagnóstico do câncer ginecológico, principalmente para as mais jovens, além de serem mais afetadas negativamente pelos cânceres ginecológicos em comparação com outros tipos de câncer e doenças crônicas, pois apesar dos efeitos adversos físicos e psicológicos diminuírem com o tempo, o impacto na vida sexual permanece por um período mais longo (Correia et al, 2020).

Desta forma, este estudo propôs como objetivo identificar os hábitos sexuais das mulheres com câncer ginecológico, submetidas ao tratamento oncológico, bem como investigar os fatores que permeiam as questões da sexualidade dessas mulheres.

\section{Metodologia}

Trata-se de uma pesquisa prospectiva, observacional, desenvolvida em um hospital de referência em oncologia, localizado no Estado do Rio de Janeiro, previamente aprovado pelo Comitê de Ética e Pesquisa. A pesquisa foi realizada entre agosto e setembro de 2018.

Participarem da pesquisa 83 pacientes selecionadas por meio de amostra não probabilística, por conveniência, sendo incluídas as portadoras de câncer ginecológico, maiores de 18 anos, internadas para realização de tratamento clínico e/ou cirúrgico e que aceitaram participar da pesquisa, após assinatura no Termo de Consentimento Livre e Esclarecido (TCLE), conforme resolução 466/12 (Conselho Nacional de Saúde, 2012). Foram excluídas as pacientes analfabetas, internadas na unidade de terapia intensiva, e as que apresentaram alterações cognitivas ou níveis de consciência rebaixados, podendo comprometer as informações colhidas.

Para a coleta dos dados foi elaborado um instrumento, tipo questionário de múltipla escolha contendo as características sociodemográficas e clínicas com 12 variáveis: idade, cor, grau de instrução, estado civil, tipo de câncer, estadiamento, tratamento antineoplásico, motivo da internação atual, sinais e sintomas clínicos, gestas, menarca e sexarca, e cinco variáveis direcionadas às questões sobre os hábitos sexuais de mulheres com câncer ginecológico após o diagnóstico, com as seguintes informações: vive com companheiro; vida sexual ativa; frequência da atividade sexual; limitações para manutenção da atividade sexual; a sexualidade alterou a qualidade de vida. O questionário foi preenchido pela participante, durante o período da internação, sob a orientação de um componente da pesquisa o qual não possuía vínculo com o setor, a fim não caracterizar uma relação de poder frente às pacientes e minimizar viés de resposta e respeitando o anonimato dos usuários.

Os dados foram tabulados e analisados no SPSS, por meio de estatística descritiva com apresentação de média e desvio padrão para as variáveis contínuas e frequência absoluta e relativa para variáveis categóricas.

\section{Resultados}

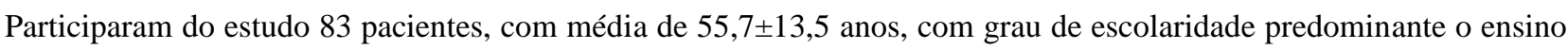
fundamental incompleto (32\%), na sua maioria casadas $(33,73 \%)$ e solteiras $(33,73 \%)$, com 3 gestas ou mais $(60,2 \%)$, pelo menos $30,1 \%$ delas apresentaram algum tipo de aborto, 54,2\% com menarca entre 11 e 13 anos, conforme pode-se observar na Tabela 1. 
Research, Society and Development, v. 11, n. 2, e49111226116, 2022

(CC BY 4.0) | ISSN 2525-3409 | DOI: http://dx.doi.org/10.33448/rsd-v11i2.26116

Tabela1: Dados sociodemográficos das pacientes participantes.

\begin{tabular}{|c|c|c|c|c|}
\hline Características & Média & $\mathrm{DP}$ & Pacientes $(\mathrm{n}=83)$ & $\%$ \\
\hline Idade & 55,7 & $\pm 13,5$ & & \\
\hline \multirow{5}{*}{ Cor/raça } & Branco & & 0 & 0 \\
\hline & Preto & & 40 & 48,2 \\
\hline & Pardo & & 27 & 32,5 \\
\hline & Amarelo & & 13 & 15,7 \\
\hline & Indígena & & 2 & 2,4 \\
\hline \multirow{6}{*}{ Estado Civil } & Solteiro & & 28 & 33,7 \\
\hline & Casado & & 28 & 33,7 \\
\hline & Divorciado & & 10 & 12 \\
\hline & União estável & & 2 & 2,4 \\
\hline & Viúva & & 9 & 10,8 \\
\hline & Outro & & 6 & 7,2 \\
\hline \multirow{7}{*}{ Número de Gesta } & 0 nenhum & & 7 & 8,4 \\
\hline & $1 \mathrm{Hum}$ & & 13 & 15,7 \\
\hline & 2 Dois & & 13 & 15,7 \\
\hline & 3 Três & & 22 & 26,5 \\
\hline & 4 Quatro & & 11 & 13,3 \\
\hline & 5 Cinco & & 9 & 10,8 \\
\hline & $>5$ maior que cinco & & 8 & 9,6 \\
\hline \multirow{4}{*}{ Aborto } & Não teve & & 58 & 69,9 \\
\hline & Espontâneo & & 15 & 18,1 \\
\hline & Provocado & & 6 & 7,2 \\
\hline & Natimorto & & 4 & 4,8 \\
\hline & Entre $8-10$ anos & & 6 & 7,2 \\
\hline & Entre $11-13$ anos & & 45 & 54,2 \\
\hline \multirow[t]{5}{*}{ Menarca } & Entre $14-16$ anos & & 29 & 34,9 \\
\hline & Acima de 16 anos & & 1 & 1,2 \\
\hline & Não sei & & 2 & 2,4 \\
\hline & Abaixo de 15 anos & & 6 & 7,2 \\
\hline & Entre $15-20$ anos & & 52 & 62,7 \\
\hline \multirow[t]{5}{*}{ Sexarca } & Entre $21-25$ anos & & 16 & 19,3 \\
\hline & Entre $26-30$ anos & & 2 & 2,4 \\
\hline & Mais de 30 anos & & 1 & 1,2 \\
\hline & Nunca tive relações sexuais & & 1 & 1,2 \\
\hline & Não sei & & 5 & 6 \\
\hline
\end{tabular}

Fonte: Autores do estudo. 
Com relação ao câncer ginecológico, observa-se na Tabela2 que as maiores incidências foram para o câncer de colo de útero $(33,73 \%)$, corpo de útero $(30,1 \%)$ e ovário $(28,9 \%)$, a maioria delas teve diagnóstico em estadiamento avançado, > IIb, III e IV (34,8\%), sendo que 30,1\% delas não tinham estadiamento ainda definido, 47\% diagnosticadas há menos de 6 meses e 38,6\% há mais de 1 ano. Metade das pacientes estava internada para cirurgias eletivas (50,6\%), e os outros 48,2\% das internações foram por urgência clínica, sendo que 9,6\% ainda aguardavam definição de tratamento, e as demais relacionadas a sequelas tardias ao tratamento antineoplásico, com predomínio de terapia combinada de quimioterapia, radioterapia e braquiterapia $(13,3 \%)$.

No que concerne os sinais e sintomas clínicos apresentados pelas pacientes analisadas no momento da entrevista, o mais prevalente foi o sangramento vaginal acometendo 32,5\% das pacientes, seguido por náusea $(30,1 \%)$ e vômitos (22,9\%).

Tabela 2: Dados clínicos das pacientes participantes:

\begin{tabular}{|c|c|c|c|}
\hline & & Pacientes $(n=83)$ & $\%$ \\
\hline \multirow{4}{*}{ Tipos de Câncer } & Vulva & 4 & 4,8 \\
\hline & Colo de útero & 28 & 33,7 \\
\hline & Corpo de útero (endométrio) & 25 & 30,1 \\
\hline & Ovário & 24 & 28,9 \\
\hline \multirow{12}{*}{ Estadiamento } & $\mathrm{NE}$ & 25 & 30,1 \\
\hline & I & 3 & 3,6 \\
\hline & IA & 2 & 2,4 \\
\hline & IB & 2 & 2,4 \\
\hline & II & 12 & 14,5 \\
\hline & IIB & 6 & 7,2 \\
\hline & III & 8 & 9,6 \\
\hline & IIIA & 0 & 0,0 \\
\hline & IIIB & 8 & 9,6 \\
\hline & IIIC & 1 & 1,2 \\
\hline & IV & 6 & 7,2 \\
\hline & SR & 8 & 9,6 \\
\hline \multirow{10}{*}{ Tratamento } & Sem tratamento & 8 & 9,6 \\
\hline & Cirurgia & 42 & 50,6 \\
\hline & Quimioterapia & 1 & 1,2 \\
\hline & Radioterapia & 5 & 6,0 \\
\hline & Quimioterapia+ Radioterapia & 2 & 2,4 \\
\hline & Quimioterapia+ Radioterapia+ Braquiterapia & 11 & 13,3 \\
\hline & Cirurgia+ Radioterapia+ Braquiterapia & 3 & 3,6 \\
\hline & Cirurgia+ Quimioterapia & 8 & 9,6 \\
\hline & Radioterapia+ Braquiterapia & 1 & 1,2 \\
\hline & Outro & 2 & 2,4 \\
\hline
\end{tabular}


Research, Society and Development, v. 11, n. 2, e49111226116, 2022

(CC BY 4.0) | ISSN 2525-3409 | DOI: http://dx.doi.org/10.33448/rsd-v11i2.26116

\begin{tabular}{lllll}
\hline \multirow{2}{*}{ Motivo da internação } & Urgência & 40 & 48,2 \\
\cline { 2 - 5 } & Eletiva & 42 & 50,6 \\
\hline \multirow{3}{*}{ Complicações mais comum } & Sangramento & 27 & 32,5 \\
\cline { 2 - 5 } & Náusea & 25 & 30,1 \\
\cline { 2 - 5 } & Vômito & 19 & 22,9 \\
\cline { 2 - 5 } & Outros & 12 & 14,5 \\
\hline
\end{tabular}

Fonte: Autores do estudo.

A Tabela 3 expõe sobre os hábitos sexuais pós diagnóstico, 75,9\% declaram não ter vida sexual ativa, estando 39,3\% atribuído à ausência de parceiro, 20,6\% por dor, 19,9\% por perda da libido e 6,3\% por medo do ato após o diagnóstico. Apenas 77 mulheres responderam quanto ao impacto da sexualidade na sua qualidade de vida, das quais $28,6 \%$ disseram que sua sexualidade pós diagnóstico interferiram na sua qualidade de vida.

Tabela 3: Hábitos sexuais pós diagnóstico.

\begin{tabular}{|c|c|c|c|}
\hline & & Pacientes $(n=83)$ & $\%$ \\
\hline \multirow{2}{*}{ Vive com companheiro } & Sim & 40 & 48,2 \\
\hline & Não & 43 & 51,8 \\
\hline \multirow{2}{*}{ Vida sexual ativa } & Sim & 19 & 22,9 \\
\hline & Não & 64 & 77,1 \\
\hline \multirow{6}{*}{ Frequência da atividade sexual } & $1 \mathrm{vez} / \mathrm{mês}$ & 6 & 31,6 \\
\hline & 2 vezes/mês & 3 & 15,8 \\
\hline & $1 \mathrm{vez} / \mathrm{semana}$ & 2 & 10,5 \\
\hline & 2 vezes/semana & 3 & 15,8 \\
\hline & 3 vezes/semana & 4 & 21,0 \\
\hline & $>3$ vezes/semana & 1 & 5,3 \\
\hline \multirow{8}{*}{ Limitações para manutenção da atividade sexual } & Medo & 5 & 6,0 \\
\hline & Dispaurenia & 10 & 12,1 \\
\hline & Vergonha do corpo & 1 & 1,2 \\
\hline & Opção do marido & 2 & 2,4 \\
\hline & Perda da libido & 14 & 16,9 \\
\hline & Limitação clínica & 6 & 7,2 \\
\hline & Ausência de parceiro & 30 & 36,1 \\
\hline & Não respondeu & 15 & 18,1 \\
\hline \multirow{3}{*}{ A sexualidade alterou a qualidade de vida } & Sim & 22 & 26,5 \\
\hline & Não & 55 & 66,3 \\
\hline & Não respondeu & 6 & 7,2 \\
\hline
\end{tabular}




\section{Discussão}

A função sexual é um aspecto importante da qualidade de vida que pode influenciar o ajuste e o enfrentamento das pacientes sobreviventes do câncer ginecológico ${ }^{6}$. A complexidade da sexualidade da mulher envolve aspectos relacionados a sua atribuição social, cultural e emocional, portanto a integralidade da mulher não pode deixar de ser considerada no plano terapêutico (Sears et al, 2018).

O câncer ginecológico e o tratamento causam problemas importantes na vida das mulheres acometidas, revelando efeitos negativos na qualidade de vida, e estão atrelados ao funcionamento sexual prejudicado, o qual está relacionado à autoimagem corporal, a sua feminilidade como cuidadora e provedora do lar, e a sua fertilidade (La Rosa, et al, 2020).

No contexto sociodemográfico, o acesso ao diagnóstico precoce esbarra em barreiras organizacionais e desigualdades sociais, econômicas, culturais e raciais (Lopes \& Ribeiro, 2019) O diagnóstico tardio compromete a sobrevida das mulheres, uma vez que a progressão da doença resulta em tratamentos mais agressivos, muitas vezes associados a duas ou mais modalidades terapêuticas, pode propiciar um resultado menos efetivo, aumentando o comprometimento físico e emocional da mulher e de sua rede de apoio, e pode impactar negativamente na esfera da sexualidade e na qualidade de vida dessas pacientes (La Rosa, et al, 2020).

No presente estudo a maioria das mulheres teve diagnóstico em estadiamento avançado, > IIb (34,8\%), 32\% das mulheres tinham o ensino fundamental incompleto, com predominância de pacientes de raça não branca e corrobora com os achados de Silva et al (2018) que apresentaram em seu estudo mulheres de raça/cor não branca (91,75\%) e com até o ensino fundamental incompleto (44,32\%). Para Madeiro et al (2016), as condições socioeconômicas são determinantes nas condições de saúde. Mulheres com baixo grau de instrução têm maior risco de desenvolver o câncer ginecológico e maior risco de diagnóstico avançado desses tumores (Correia et al, 2018).

Isso se justifica porque embora o Ministério da Saúde estabeleça diretrizes para a prevenção e rastreamento do câncer de colo de útero, o estadiamento avançado demonstra falhas na triagem e detecção precoce dessas neoplasias e pode estar relacionado com a falta de informação e acesso aos serviços de saúde (Martins et al, 2017). Desta forma, mulheres com mais idade têm sido mais frequentemente diagnosticadas em estadiamentos mais avançados do que mulheres mais jovens. As pacientes do estudo em tela tinham idade média $55,7 \pm 13,5$ anos e foram diagnosticadas em fase avançada, $34,8 \%$ dos casos apresentam estadiamento maior que IIb.

A considerar os fatores psicológicos relacionados a sexualidade da mulher, destaca-se que a alteração de imagem corporal e da feminilidade ligados a perda de órgãos como útero e/ou ovários, podem gerar um sentimento de insegurança, insatisfação no relacionamento e frustrações diante de sua imagem corporal, interferindo em suas relações pessoais que podem levar a perda da libido e dificuldades de excitação (Fernández-Cacho \& Ayesa-Arriola, 20190; Pimetel et al, 2020).

As mulheres do estudo apresentam características sugestivas de uma vida sexual prévia ao diagnóstico hiperativa e com início precoce, visto que 54,2\% das pacientes entraram em menarca entre 11 e 13 anos e 60,2\% relataram pelo menos 3 gestas ou mais, pelo menos 30,1\% delas apresentaram algum tipo de aborto, achado semelhante foi relatado por Maranhão et al (2017) que observaram que quanto mais precoce se dava o início das relações sexuais, maior era a repercussão negativa sobre a vida reprodutiva e sexual, com maiores números de gestas.

No pós diagnóstico, nota-se uma baixa incidência de mulheres sexualmente ativas $(22,89 \%)$, esse número representa metade do achado relatado no estudo de Grimm et al (2015) em que apenas 41.3\% das mulheres após tratamento de câncer ginecológico eram sexualmente ativas.

A ausência de parceiros pode justificar uma baixa incidência das mulheres em manter ativa a prática da relação sexual após tratamento oncológico ginecológico, foi observado que 36,1\% das mulheres eram casadas ou viviam em união estável, e 
33,7\% eram solteiras. Além disso, há rejeição dos companheiros, que mesmo presentes, por vezes não conseguem vê-las como uma parceira sexual, interferindo na perda da intimidade (Bae \& Park, 2016).

O apoio psicossexual deve ir além do funcionamento sexual físico e deve levar em consideração aspectos como identificar sofrimento sexual, a satisfação no relacionamento e a perspectiva do parceiro (Vermeer et al., 2016). As mulheres do estudo relataram como principais limitações para a manutenção da prática sexual, a ausência de parceiros (36,14\%), a perda da libido $(16,87 \%)$ e a dispauremia $(12,05 \%)$, além disso, embora numa baixa incidência, algumas mulheres referiram ter vergonha do corpo $(1,2 \%)$ e a rejeição do marido $(2,4 \%)$, repercutindo negativamente na sua vida conjugal. A participação do parceiro na terapêutica da função sexual é fundamental para o sucesso em quebrar os tabus, vencer os medos e respeitar as limitações inerentes da sexualidade da mulher pós tratamento de câncer ginecológico (Abbott-Anderson et al, 2020).

Com o crescente número de mulheres em tratamento para o câncer ginecológico, a atenção à sexualidade é crucial para o cuidado integral dessas mulheres. Nesse sentido, a sexualidade é, segundo a Organização Mundial de Saúde, um dos indicadores de qualidade de vida. Considera-se, assim, que a avaliação da qualidade de vida relacionada à saúde e a sexualidade de mulheres com esse tipo de câncer é de suma importância, uma vez que não envolve apenas os fatores orgânicos dos órgãos genitais, mas também aspectos mentais, corporais e sociais, capazes de influenciar no bem-estar físico e mental afetados pelos agravos do tratamento (Albers et al, 2020).

Parece que as preocupações sexuais físicas têm recebido mais atenção do que as preocupações psicológicas e sociais, mulheres sobreviventes de câncer ginecológico mostram-se pouco esclarecidas quanto às possibilidades de tratamento para resolver suas questões sexuais (Pinheiro et al, 2019).

A pesquisa teve como principal limitação utilizar apenas um cenário, reduzindo a um ponto de vista de uma população da rede pública de saúde sobre os fatores que interferem na sexualidade dessas mulheres, não refletindo as repercussões de mulheres de outras condições socioeconômicas e culturais de um modo geral.

\section{Considerações Finais}

Os resultados mostraram características como baixo grau de escolaridade, elevado número de gestas e elevadas ocorrências de abortos como fatores que podem estar relacionados ao risco de câncer ginecológico, bem como as elevadas prevalências de estadiamento avançado e internações por urgências clinicas apontam para a necessidade de novas estratégias na implementação das políticas de saúde no que diz respeito ao diagnóstico precoce e ao monitoramento e controle clinico pós tratamento antineoplásico.

A situação conjugal mostra-se relevante na função sexual das mulheres, mas percebe-se o importante impacto dos sintomas de dor e perda de libido na sexualidade dessas mulheres.

Há ainda uma necessidade de maior exploração dessa temática para instruções não só das pacientes e acompanhantes, mas, sobretudo dos profissionais da equipe de saúde que irão conduzir esta abordagem.

Contudo esses resultados sugerem que é cada vez mais importante o conhecimento dos aspectos psicossociais envolvidos nos cuidados de pacientes com câncer ginecológico e talvez um estudo qualitativo poderia elucidar melhor as estratégias de enfretamento da doença e trazer uma visão mais integrada dos cuidados dessas mulheres que possa contribuir para uma melhor qualidade de vida.

\section{Referências}

Abbott-Anderson, K. et al. (2020). Adjusting to sex and intimacy: Gynecological cancer survivors share about their partner relationships. Journal of women \& aging, 32(3), 329-348. https://doi.org/10.1080/08952841.2019.1591888

Albers, L. F. et al. (2020). Discussing sexuality in cancer care: towards personalized information for cancer patients and survivors. Supportive care in cancer, 28(9), 4227-4233. https://doi.org/10.1007/s00520-019-05257-3 
Research, Society and Development, v. 11, n. 2, e49111226116, 2022

(CC BY 4.0) | ISSN 2525-3409 | DOI: http://dx.doi.org/10.33448/rsd-v11i2.26116

American cancer society (2019). Cancer facts \& figures 2019. American Cancer Society.

Bae, H. \& Park, H. (2016). Sexual function, depression, and quality of life in patients with cervical cancer. Supportive care in cancer, 24(3), 1277-1283. https://doi.org/10.1007/s00520-015-2918-z

Conselho nacional de saúde (2020). Resolução n. 466, de 12 de dezembro de 2012. https://conselho.saude.gov.br/resolucoes/2012/Reso466.pdf.

Correia, R. A. et al. (2018). Quality of life after treatment for cervical cancer. Escola Anna Nery, 22(4). https://doi.org/10.1590/2177-9465-EAN-2018-0130

Correia, R. A. et al. (2020). Disfunção sexual após tratamento para o câncer do colo do útero. Revista da Escola de Enfermagem da USP, 54. https://doi.org/10.1590/S1980-220X2019029903636

Fernández-Cacho, L. M \& Ayesa-Arriola, R. (2019). Quality of life, pain and anxiety in patients with nephrostomy tubes.. Revista Latino-Americana de Enfermagem, 27. https://doi.org/10.1590/1518-8345.3039.3191

Grimm, D. et al. (2015). Sexual Activity and Function in Patients With Gynecological Malignancies After Completed Treatment. Int J Gynecol Cancer, 25(6), 1134-1341. http://dx.doi.org/10.1097/IGC.0000000000000468

Inca (2020). Estimativa/2020. Incidência de Câncer no Brasil.

La Rosa, V. L. et al. (2020). Fertility preservation in women affected by gynaecological cancer: the importance of an integrated gynaecological and psychological approach. ecancermedicalscience, 14 . https://dx.doi.org/10.3332\%2Fecancer.2020.1035

Lopes, V. A. S. \& Ribeiro, J. M. (2019). Fatores limitadores e facilitadores para o controle do câncer de colo de útero: uma revisão de literatura. Ciência \& Saúde Coletiva, 24, 3431-3442. https://doi.org/10.1590/1413-81232018249.32592017

Madeiro, A. et al. (2016). Tendências da mortalidade por câncer do colo do útero no Piaú, 2000-2011. Cadernos Saúde Coletiva, 24(3), 282-285. https://doi.org/10.1590/1414-462X201600030026

Maranhão, T. A. et al. (2017). Repercussão da iniciação sexual na vida sexual e reprodutiva de jovens de capital do Nordeste brasileiro. Ciência \& Saúde Coletiva, 22, 4083-4094. https://doi.org/10.1590/1413-812320172212.16232015

Martins, J., et al. (2017). Factors associated with changes in vaginal length and diameter during pelvic radiotherapy for cervical cancer. Archives of gynecology and obstetrics, 296(6), 1125-1133. https://doi.org/10.1590/1806-93042018000400002

Pimentel, N. B. L. et al. (2020). O câncer do colo uterino e o impacto psicossocial da radioterapia pélvica: revisão integrativa. Research, Society and Development, 9(10), e6489109052-e6489109052. https://doi.org/10.33448/rsd-v9i10.9052

Pinheiro, C. C. et al. (2019). Qualidade de vida após o câncer ginecológico. Revista Brasileira de Sexualidade Humana, 30(1). https://doi.org/10.35919/rbsh.v30i1.67

Sears, C. S. et al. (2018). A comprehensive review of sexual health concerns after cancer treatment and the biopsychosocial treatment options available to female patients. European journal of cancer care, 27(2), e12738. https://doi.org/10.1111/ecc.12738

Silva, R. C. G. et al. (2018). Perfil de mulheres com câncer de colo do útero atendidas para tratamento em centro de oncologia. Revista Brasileira de Saúde Materno Infantil, 18(4), 695-702. https://doi.org/10.1590/1806-93042018000400002

Vermeer, W. M. et al. (2016). Cervical cancer survivors' and partners' experiences with sexual dysfunction and psychosexual support. Supportive Care in Cancer, 24(4), 1679-1687. https://doi.org/10.1007/s00520-015-2925-0 